Article

\title{
Vaccination with Consensus H7 Elicits Broadly Reactive and Protective Antibodies against Eurasian and North American Lineage H7 Viruses
}

\author{
Gendeal M. Fadlallah ${ }^{1}$, Fuying Ma ${ }^{1}\left(\right.$, Zherui Zhang ${ }^{2}$, Mengchan Hao ${ }^{2,3}$, Juefu Hu ${ }^{2,3}$, \\ Mingxin Li ${ }^{2}$, Haizhou Liu 2,3 (D), Biling Liang ${ }^{2,3,4}$, Yanfeng Yao ${ }^{5}$, Rui Gong ${ }^{2,4}$, Bo Zhang ${ }^{2,4}$, \\ Di Liu ${ }^{2,3,4}$ and Jianjun Chen $2,3,4, *$ (D) \\ 1 Department of Biotechnology, Key Laboratory of Molecular Biophysics of MOE, College of Life Science and \\ Technology, Huazhong University of Science and Technology, Wuhan 430074, China; \\ Gend_178@hotmail.com (G.M.F.); mafuying@hust.edu.cn (F.M.) \\ 2 CAS Key Laboratory of Special Pathogens and Biosafety, Chinese Academy of Sciences, Wuhan 430071, \\ China; zherui4138@163.com (Z.Z.); hmc@wh.iov.cn (M.H.); hujuefu2018@163.com (J.H.); \\ 2009limingxin@163.com (M.L.); liuhz@wh.iov.cn (H.L.); liangbl@wh.iov.cn (B.L.); gongr@wh.iov.cn (R.G.); \\ zhangbo@wh.iov.cn (B.Z.); liud@wh.iov.cn (D.L.) \\ 3 National Virus Resource Center, Chinese Academy of Sciences, Wuhan 430071, China \\ 4 University of Chinese Academy of Sciences, Beijing 100000, China \\ 5 National Biosafety Laboratory, Wuhan Institute of Virology, Chinese Academy of Sciences, Wuhan 430071, \\ China; yaoyf@wh.iov.cn \\ * Correspondence: chenjj@wh.iov.cn; Tel.: +86-27-87198739
}

Received: 22 February 2020; Accepted: 16 March 2020; Published: 23 March 2020

\begin{abstract}
H7 subtype avian influenza viruses have caused outbreaks in poultry, and even human infection, for decades in both Eurasia and North America. Although effective vaccines offer the best protection against avian influenza viruses, antigenically distinct Eurasian and North American lineage subtype $\mathrm{H} 7$ viruses require the development of cross-protective vaccine candidates. In this study, a methodology called computationally optimized broadly reactive antigen (COBRA) was used to develop four consensus $\mathrm{H} 7$ antigens (CH7-22, $\mathrm{CH} 7-24, \mathrm{CH} 7-26$, and $\mathrm{CH} 7-28$ ). In vitro experiments confirmed the binding of monoclonal antibodies to the head and stem domains of cell surface-expressed consensus HAs, indicating display of their antigenicity. Immunization with DNA vaccines encoding the four antigens was evaluated in a mouse model. Broadly reactive antibodies against H7 viruses from Eurasian and North American lineages were elicited and detected by binding, inhibition, and neutralizing analyses. Further infection with Eurasian H7N9 and North American H7N3 virus strains confirmed that $\mathrm{CH} 7-22$ and $\mathrm{CH} 7-24$ conferred the most effective protection against hetero-lethal challenge. Our data showed that the consensus $\mathrm{H7}$ vaccines elicit a broadly reactive, protective response against Eurasian and North American lineage $\mathrm{H} 7$ viruses, which are suitable for development against other zoonotic influenza viruses.
\end{abstract}

Keywords: COBRA; influenza; consensus H7; broadly reactive; mice

\section{Introduction}

H7 subtype avian influenza virus has caused outbreaks in poultry, and even human infection, for decades. Both North American and Eurasian lineages of H7 viruses have been associated with human infection [1], and the global distribution of this subtype has affected poultry in Europe, America, Asia, and Oceania [2]. To date, human infections have been caused by the poorly pathogenic H7 subtypes, H7N2, H7N3, H7N4, H7N7, and H7N9, as well as the highly pathogenic H7N3 and 
H7N7 [2-5]. Before 2003, less than 20 sporadic cases of human infection with H7 viruses were reported in Europe and America [1]. In 2003, outbreaks of HPAI H7N7 occurred in poultry of several European countries, and 86 poultry workers as well as three of their family members were infected with this subtype in The Netherlands [5]. Among them, almost all infected people developed mild-moderate conjunctivitis and one person died from pneumonia and acute respiratory distress syndrome [5]. This outbreak represented the first H7 avian influenza outbreak in humans. H7N9 caused another outbreak in 2013 in China [4,6]. The viruses most likely emerged in the Yangtze River Delta region of China and disseminated widely throughout the country $[7,8]$. Since its emergence in 2013, the H7N9 virus has been circulating in domestic poultry in China and caused five epidemic waves of human infections, and it has evolved continually and substantially [9-13]. As of December 2019, 1568, laboratory-confirmed cases of human infection with H7N9 viruses, including at least 616 deaths, have been reported to the WHO [14].

To date, H7 influenza viruses have not caused a pandemic, but have the potential. Vaccination is an effective strategy to prevent and control enzootic influenza infection. However, antigenically distinct Eurasian and North American lineage subtype H7 viruses require the development of appropriate vaccine candidates. Currently available influenza vaccines mainly provide hemagglutinin strain-specific protection, but rarely provide cross-protection against divergent strains. It is therefore particularly important to develop an intra- and inter-subtype universal vaccine. In recent years, a methodology called computationally optimized broadly reactive antigen (COBRA) was used to design intra-subtype universal influenza vaccines [15]. COBRA employs multiple rounds of layered consensus building to generate influenza vaccine HA immunogens [16]. This strategy has been used to develop broadly protective vaccine candidates against various influenza subtypes in animal models, including enzootic highly pathogenic H5 [15], seasonal circulating H1 [17], and H3 viruses [18]. The success in preclinical data highly suggests that the COBRA strategy is flexible to develop vaccines against other subtypes of influenza viruses.

DNA vaccines constitute a powerful alternative to conventional vaccines because they can induce both humoral and cellular immune responses. With DNA vaccinations, because the encoded protein is synthesized in its native form inside the host cell, the antibody responses induced in mice by consensus H7 DNA vaccination in our study reflected the real immunogenicity of the antigen in mice. In this study, four consensus $\mathrm{H7}$ immunogens were generated based on the COBRA approach. Immunization with DNA vaccines encoding the four antigens were then evaluated in a mouse model. The mice elicited broadly reactive antibodies against $\mathrm{H} 7$ viruses from Eurasian and North American lineages. Further infection with Eurasian H7N9 and North American H7N3 virus strains confirmed that two consensus antigens (CH7-22 and CH7-24) conferred the most effective protection against hetero-lethal challenge.

\section{Materials and Methods}

\subsection{Ethics Statement}

This study was reviewed and permitted by the Ethics Committee of the Wuhan Institute of Virology, Chinese Academy of Sciences. Animal experiments were carried out in an animal biosafety level 2 facility. The experiments were conducted under the Chinese national guidelines of ethics and policies for the care of laboratory animals and were certified by the Welfare and Ethical Review Board of Wuhan Institute of Virology's Institutional Animal Care and Use Committee.

\subsection{Cells and Viruses}

Madin Darby Canine Kidney (MDCK) and BHK-21 cells were obtained from the National Virus Resource Center, Wuhan Institute of Virology, Chinese Academy of Sciences, and cultured in Dulbecco's modified Eagle's medium (DMEM) supplemented with 10\% fetal bovine serum. The H7N9 virus used in the animal experiments was NIBRG-267 (H7N9), a vaccine strain from the National Institute for Biological Standards and Control (NIBSC) and adapted to mice [19]. H7N7 [A/Phalacrocorax 
carbo/Hubei/HH179/2013 (H7N7)] was isolated from wild waterfowl and described previously [20]. H7N3 [A/chicken/BC/CN006/2004 (H7N3) (rBC04/H7N3)] and H7N1 [A/Rhea/North Carolina/39482/93 (H7N1) (rNC93/H7N1)] with two surface genes of their own and six internal genes from A/Puerto Rico/8/34 were prepared in our laboratory [21]. H7N9 and H7N7 strains represent Eurasian lineage viruses, and H7N3 and H7N1 strains were North American viruses. H7N9, H7N7, H7N3, and H7N1 viruses were inoculated into 10-day-old SPF embryonated chicken eggs to propagate. The median tissue culture infective dose $\left(\mathrm{TCID}_{50}\right)$ of the viruses was determined with eight replicates in fresh medium containing $1 \mu \mathrm{g} / \mathrm{mL}$ TPCK-trypsin (Sigma-Aldrich, St. Louis, USA).) [22].

\subsection{Antigenic Construction and Characterization}

Human H7 HA protein sequences $(n=1502)$ were downloaded from the NCBI influenza virus resource and GISAID (Global Initiative on Sharing Avian Influenza Data) database until early 2017. A multiple alignment analysis was conducted and a maximum-likelihood phylogenic tree was generated using MEGA7 software. Consensus generation of each round was performed as described previously [18]. Briefly, for each round of consensus generation, multiple alignment was performed and the most common residue was obtained and yielded the consensus sequence. Multiple rounds of consensus assembly were layered to yield primary and secondary consensus sequences. The last outcome of amino acid sequences was applied to COBRA. Four H7 HA protein sequences were generated and named $\mathrm{CH} 7-22, \mathrm{CH} 7-24, \mathrm{CH} 7-26$, and $\mathrm{CH} 7-28$. A phylogenetic tree was inferred from hemagglutinin amino acid sequences using the maximum likelihood method, and groupings of the four $\mathrm{H} 7$ antigens in trees were identified using Figtree software. Furthermore, to determine whether the four sequences could form a three-dimensional conformation, homology models of CH7-22, CH7-24, CH7-26, and CH7-28 were constructed in the SWISS-MODEL webserver. The antigenic sites of H7 HA COBRA were determined based on alignment with the A/Anhui/1/2013 (H7N9) sequence as described previously [23]. Colorization of five antigenic sites (A, B, C, D and E) in trimerized $\mathrm{H} 7$ protein was performed using PyMol.

\subsection{Gene Synthesis and Plasmid Construction}

After designing the consensus immunogens, the four consensus sequences were subjected to codon/RNA optimization to enhance gene expression in mammal cells as described previously [24,25]. The four H7 COBRA HAs were synthesized (Sangon Biotech, Shanghai, China) and cloned into the eukaryotic expression plasmid pVAX1 (Invitrogen, San Diego, CA, USA) using XhoI and EcoRI with a Kozak translation initiation sequence (GCCACC) inserted before the start codon. The plasmids were propagated in Escherichia coli DH5 $\alpha$ and purified using an E.Z.N.A. ${ }^{\circledR}$ Endo-Free Plasmid Maxi Kit (Omega Bio-tek, Inc., Norcross, GA, USA). Sequencing was performed to ensure no mutations were in the plasmid. The HA genes of A/Shanghai/02/2013 (H7N9) and A/Phalacrocorax carbo/Hubei/HH179/2013 (H7N7) were amplified and cloned into pVAX1 to generate $\mathrm{pH7N9}$ and $\mathrm{pH7N7}$, following the same method for COBRA HAs.

\subsection{In Vitro Expression of Consensus H7 HAs}

BHK-21 cells $\left(1 \times 10^{6}\right)$ were transfected with $3 \mu \mathrm{g}$ pCH7-22, pCH7-24, pCH7-26, pCH7-28, and pVAX1 (empty vector) using Lipofectamine 3000 Transfection Reagent (Invitrogen). After incubation for $5 \mathrm{~h}$, the medium was replaced with fresh medium containing $10 \%$ fetal bovine serum. At $24 \mathrm{~h}$ post-transfection, the cells were washed with PBS, fixed with $4 \%$ paraformaldehyde (pH 7.4) for 30 min, permeabilized with $0.2 \%$ Triton X-100 in PBS for $30 \mathrm{~min}$, and then stained with Hoechst 33258 for $30 \mathrm{~min}$. Indirect immunofluorescence staining was performed with monoclonal antibodies CR9114 (broad-spectrum anti-stem neutralizing antibody) [26] or P52E03 (targeting the HA head of subtype H7) [21], and then FITC-labeled goat anti-human IgG. Imaging was conducted under a fluorescence microscope (Nikon Eclipse TE2000). 


\subsection{Vaccination and Antibody Response Analysis}

In vivo electroporation was carried out according to a previously described method $[27,28]$. Six- to 8-week-old female BALB/c mice (10 mice per group) were immunized twice with a 3-week interval. Each vaccination consisted of $30 \mu \mathrm{g}$ pCH7-22, pCH7-24, pCH7-26, pCH7-28, pH7N9, and pH7N7 dissolved in $30 \mu \mathrm{L}$ Tris-EDTA buffer. Control group mice were injected with PBS. After injection into the right quadricep muscle, a pair of electrode needles at $5 \mathrm{~mm}$ apart was inserted into the muscle to cover the DNA injection sites and electric pulses were delivered using an electric pulse generator (ECM830; BTX, San Diego, CA, USA). Two weeks after the second immunization, sera were collected from four to five mice per group and treated with a receptor-destroying enzyme (RDE) (Denka Seiken, Co., Tokyo, Japan) as described previously [21]. Briefly, the sera were treated with the RDE, followed by inactivation at $56^{\circ} \mathrm{C}$ for $30 \mathrm{~min}$ and incubation with chicken erythrocytes to adsorb nonspecific agglutinins. The treated serum was tested in the following assays.

\subsubsection{Hemagglutinin Inhibition (HAI) Assay}

The sera from each group of immunized or unimmunized mice were serially diluted two-fold with PBS in a 96-well polystyrene microtiter plate with $25 \mu \mathrm{L}$ in each well. A portion of $25 \mu \mathrm{L}$ of virus suspension containing four hemagglutinin units (HAU) of H7N9, H7N7, H7N3, and H7N1 was added to each well. After incubation of the plate at room temperature for $1 \mathrm{~h}, 50 \mu \mathrm{L}$ of $0.5 \%(\mathrm{v} / \mathrm{v})$ chicken red blood cells were added to each well, and the plate was incubated at room temperature for 30 minutes. The HAI titers were determined as the highest serum dilution that completely inhibited hemagglutination.

\subsubsection{Microneutralization (MN) Assay}

Titers of neutralizing antibodies were determined as described previously [21]. Serum treated with the receptor-destroying enzyme (RDE) was diluted from 1:20 to 1:2560 by two-fold serial dilutions in culture medium (DMEM containing $100 \mathrm{U} / \mathrm{ml}$ penicillin $\mathrm{G}, 100 \mu \mathrm{g} / \mathrm{mL}$ streptomycin, and $0.5 \mu \mathrm{g} / \mathrm{mL}$ TPCK-treated trypsin). Diluted serum solutions were mixed with culture medium containing 100 TCID $_{50}$ H7N9, H7N7, H7N3, and H7N1 at room temperature for $1 \mathrm{~h}$. The virus-serum mix was then transferred to MDCK cells. Culture medium was added, and the plates were incubated for $72 \mathrm{~h}$. Endpoints were determined by the hemagglutination titer.

\subsubsection{ELISA}

An ELISA was performed using a 96-well plate (EIA plate, Costar, Richmond, VA, USA) that was first coated with H7N9, H7N7, H7N3, and H7N1 (4 HAU/well) and then incubated with serial dilutions of RDE-treated serum, followed by goat anti-mouse IgG ( $\gamma$-chain specific) (Southern Biotechnology Associates, Inc., Birmingham, UK) conjugated with HRP. The amount of chromogen produced was measured based on the absorbance at $420 \mathrm{~nm}$ using an ELISA reader (Synergy H1, Biotek, Winooski, VT, USA).

\subsection{Adaptaion of H7N3 Influenza Virus in Mice}

The mouse-adapted virus was prepared as described previously [24]. To enhance the virulence of North American lineage strain H7N3 [A/chicken/BC/CN006/2004 (H7N3) (rBC04/H7N3)], we applied 10 sequential passages in mice to achieve a mouse-adapted virus. Initially, a group of three mice was anesthetized, and each mouse was inoculated intranasally with $20 \mu \mathrm{L}$ viral H7N3. At 3-5 days post-inoculation, mice were sacrificed and their trachea and lungs were removed. Tissues were washed twice in $2 \mathrm{~mL}$ phosphate-buffered saline (PBS) containing $0.1 \%$ bovine serum albumin (BSA). The bronchoalveolar washes were collected and used to infect the next batch of mice after removing cellular debris by centrifugation. Lung-to-lung passaging was repeated until the virus was lethal in mice. After 10 serial passages, mice developed clinical symptoms, including a hunched posture, 
weakness, weight loss, and ruffled fur, and the viruses exhibited high virulence in mice. The final adapted virus was harvested, aliquoted, and stored at $-80{ }^{\circ} \mathrm{C}$. The $50 \%$ mouse lethal dose $\left(\mathrm{LD}_{50}\right)$ of each stock was determined using the Reed-Muench method.

\subsection{Viral Infection and Titration}

Three weeks after a boost, mice in each group $(n=10)$ were anesthetized and challenged with the mouse-adapted H7N9 and H7N3 viruses at $10 \times \mathrm{LD}_{50}$ by intranasal administration in $20 \mu \mathrm{L}$ viral suspension. This infection caused rapid and widespread viral replication in the lungs and death of the unimmunized mice within 7 days. At 5 days post-infection, the trachea and lungs in each group $(n=4-5)$ were removed and washed twice by injecting $2 \mathrm{~mL}$ PBS containing $0.1 \%$ BSA. The bronchoalveolar wash was used for virus titration after removing cellular debris by centrifugation. The remaining mice were observed for 14 days to record survival rates and weight loss.

Lung viral titers were determined as described previously [27]. Briefly, MDCK cells were cultured in 96-well plates overnight. After $12 \mathrm{~h}$ of culture, the cells were infected with $100 \mu \mathrm{L}$ of a 1/10-dilution series of lung homogenate supernatant at $37^{\circ} \mathrm{C}$ for $1 \mathrm{~h}$. The supernatants were then replaced with $200 \mu \mathrm{L}$ serum-free Dulbecco's modified Eagle medium (DMEM) containing a cocktail of antibiotics. The cytopathic effects in the infected MDCK cells were observed daily. After incubation for 3 days, the virus titer in the supernatant was assayed by measuring hemagglutination.

\subsection{Statistical Analysis}

Viral titers were calculated by the Reed-Muench method. All data plotted with error bars are expressed as means with SD. The $p$ values were generated by analyzing data with a two-tail unpaired $t$ test using the Prism 8 program (GraphPad software, San Diego, CA, USA) or Mann-Whitney Test. The survival rate statistical analysis was performed with a Fisher's exact test.

\section{Results}

\subsection{Design of the Four Consensus H7 Proteins}

We applied the COBRA strategy to develop cross-reactive antigens against $\mathrm{H} 7$ subtype influenza viruses. Eurasian and North American lineage human $\mathrm{H} 7$ viruses were downloaded and aligned. The viruses were divided into four groups (H7N9, H7N7, H7N3, and H7N2) based on the different HA/NA combinations. The H7N9 viruses were further subdivided into five epidemic waves (Figure 1). Primary consensus amino acid sequences were derived from the three groups (H7N7, H7N3, and H7N2) and five subgroups (referred to five wave H7N9) (Figure 1). CH7-26 was generated based on the primary consensus sequences of H7N7, H7N3, and H7N2. CH7-24 was obtained from the primary consensus sequences of five wave H7N9. Second consensus amino acid sequences were derived from primary sequences (Figure 1). Then, CH7-22 was designed based on all secondary consensus sequences. CH7-28 was derived from CH7-26 sequences by introducing S136N and A143V (with signal sequences) mutations based on the analysis showing that both mutations emerged in H7N9 HA since the second wave (data not shown). 


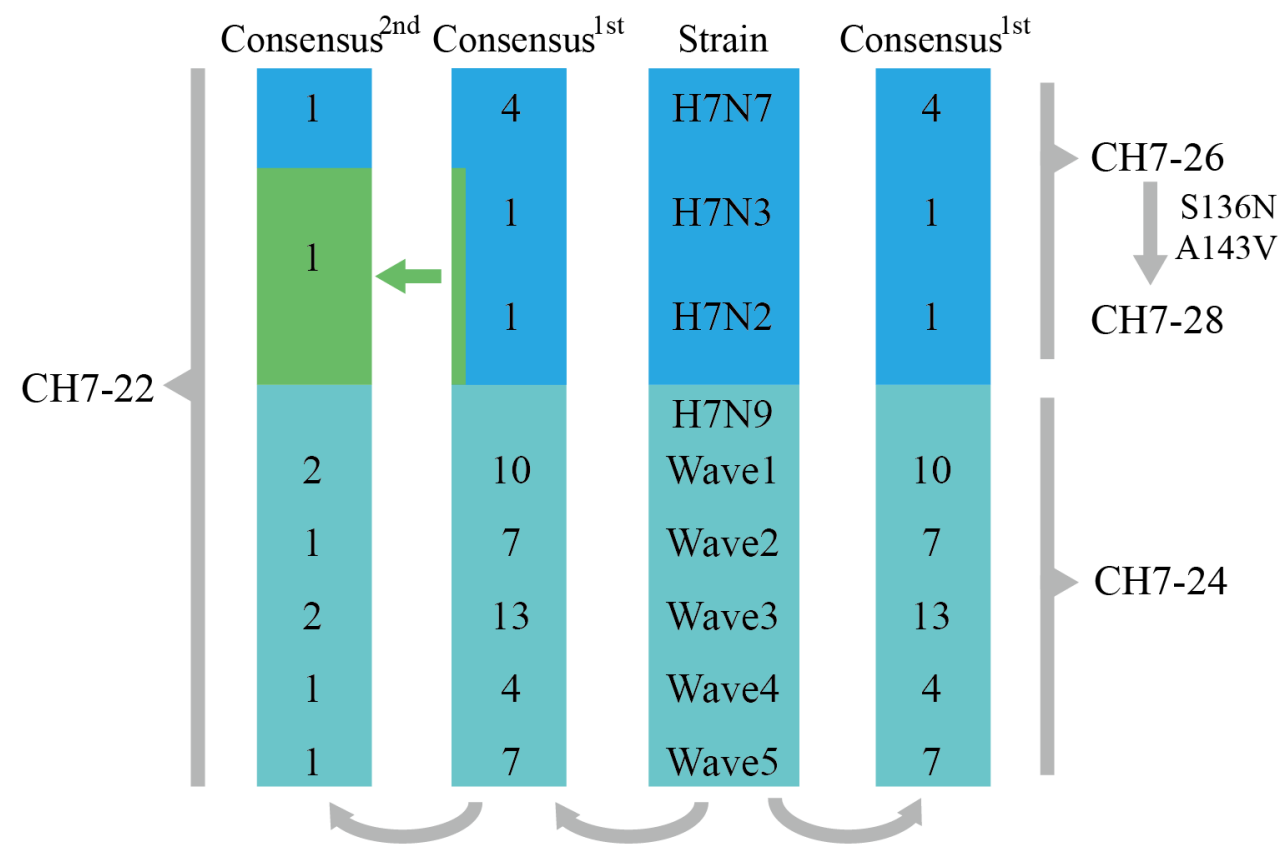

Figure 1. Schematic example of the layered consensus used for H7 COBRA design. The viruses were divided into four groups (H7N9, H7N7, H7N3, and H7N2) based on different HA/NA combinations. H7N9 viruses were further subdivided into five epidemic waves. Primary consensus amino acid sequences were derived from the three groups (H7N7, H7N3, and H7N2) and five subgroups (referred to five wave H7N9). CH7-26 was generated based on the primary consensus sequences of H7N7, H7N3, and H7N2. CH7-24 was obtained from the primary consensus sequences of five wave H7N9. Second consensus amino acid sequences were derived from primary sequences. Then, $\mathrm{CH} 7-22$ was designed based on all secondary consensus sequences. CH7-28 was derived from $\mathrm{CH} 7-26$ sequences by introducing S136N and A143V mutations. The green arrow refers to the secondary consensus, which was generated by alignment of the primary consensus of H7N3 and H7N2.

\subsection{Characterization of the Four Consensus H7 Proteins}

Sequence alignment of the four consensus antigens showed that the sequence identities ranged from $97 \%$ to $98.8 \%$. BLAST analysis of $\mathrm{CH} 7-22$, CH7-24, CH7-26, and CH7-28 sequences indicated that each sequence was unique, and the identities between any one of the four sequences and sequences deposited in NCBI range from $97 \%$ to $98 \%$ (data not shown). Phylogenetic analysis of the four consensus H7 sequences with all H7 HA proteins showed that the four COBRA were located on separate branches without grouping with any viruses of Eurasian and North American H7 lineages (Figure 2A). Using predictive structural modelling of COBRA H7 HA sequences, three-dimensional trimerized HA proteins were visualized (Figure $2 \mathrm{~B}$ ). In addition, a similar location of putative antigenic sites (A-E) of H7 HA was observed in the four COBRA sequences (Figure 2B), suggesting the consensus H7 HA maintained the native three-dimensional conformation and antigenic sites. Expression of the four COBRA H7 HA proteins was confirmed by an indirect immunofluorescence assay. BHK-21 cells transiently transfected with $\mathrm{pCH}-22$, $\mathrm{pCH}-24, \mathrm{pCH}-26$, and $\mathrm{pCH}-28$ were stained with stem-specific CR9114 or head-specific P52E03 mAbs. Binding of CR9114 or P52E03 to the four proteins was detected (Figure 3). Overall, we confirmed that the four COBRA H7 HA proteins were bound by either stem-specific or head-specific mAbs, indicating preservation of antigenic sites. 
A.

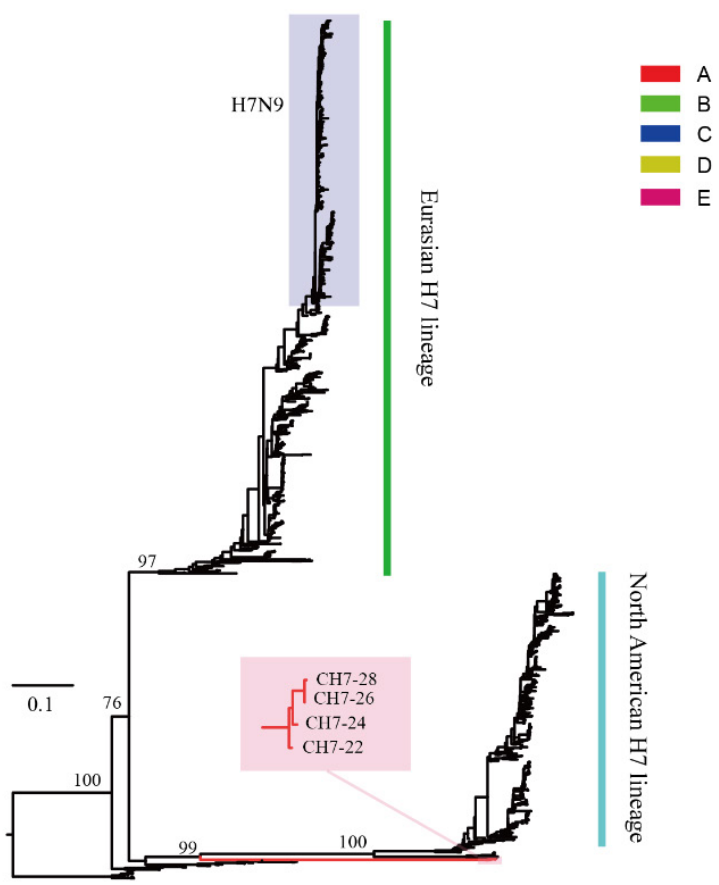

B.

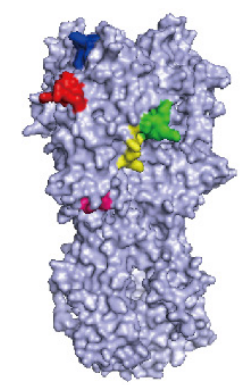

CH7-22

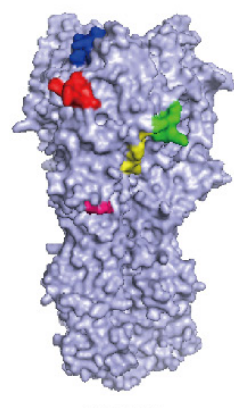

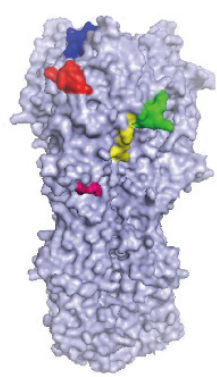

CH7-24

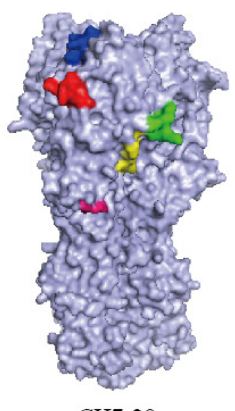

CH7-28

Figure 2. Phylogenetic analysis of the consensus H7 (A). A phylogenetic tree was inferred from the hemagglutinin amino acid sequences of $\mathrm{H} 7$ viruses $(n=1502)$ using the maximum likelihood method, and groupings of the four consensus $\mathrm{H} 7$ antigens in trees were identified using Figtree software. The four consensus H7 sequences with all H7 HA proteins showed that the four consensus H7 sequences (red branch) were located on separate branches without grouping with any viruses of Eurasian and North American H7 lineages. Schematic of the three-dimensional structure of four trimerized consensus $\mathrm{H7}$ proteins (B). After four consensus $\mathrm{H7}$ sequences were constructed, homology models were created for each one using the SWISS-MODEL webserver. The antigenic sites of consensus H7 HA were identified by alignment with the A/Anhui/1/2013 (H7N9) sequence. The A site is shown in red, the $B$ site in green, the $C$ site in blue, the $\mathrm{D}$ site in yellow, and the $\mathrm{E}$ site in medium violet-red. 


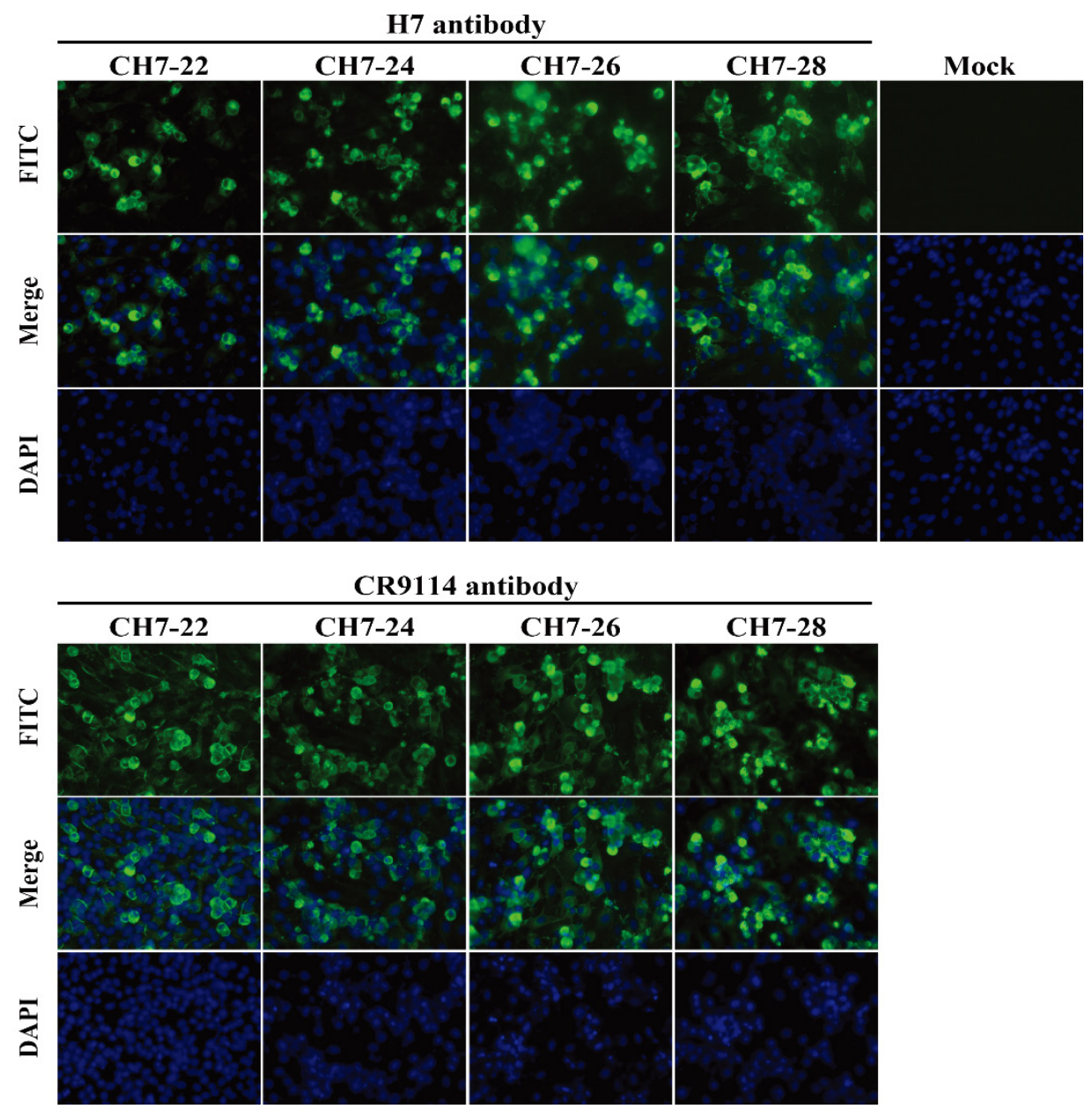

Figure 3. In vitro expression of consensus H7 HAs. BHK-21 cells $\left(1 \times 10^{6}\right)$ were transfected with $3 \mu \mathrm{g}$ pCH7-22, pCH7-24, pCH7-26, pCH7-28, and pVAX1 (empty vector) using Lipofectamine 3000 Transfection Reagent (Invitrogen). After incubation for $5 \mathrm{~h}$, the medium was replaced with fresh medium containing $10 \%$ fetal bovine serum. At $24 \mathrm{~h}$ post-transfection, the cells were washed with PBS, fixed with $4 \%$ paraformaldehyde ( $\mathrm{pH} 7.4$ ) for $30 \mathrm{~min}$, permeabilized with $0.2 \%$ Triton X-100 in PBS for $30 \mathrm{~min}$, and then stained with Hoechst 33258 for $30 \mathrm{~min}$. Indirect immunofluorescence staining was performed with monoclonal antibodies CR9114 (broad-spectrum anti-stem neutralizing antibody) or P52E03 (targeting the HA head of subtype H7) and then FITC-labeled goat anti-human IgG. Imaging was conducted under a fluorescence microscope (Nikon Eclipse TE2000).

\subsection{DNA Vaccines Encoding Consensus H7 Proteins Elicit Broadly Reactive Antibody Responses in Mice}

Upon verifying expression of all four consensus $\mathrm{H7}$ constructs, we first examined the capacity of these immunogens to induce immune responses in mice against antigenic and genetically diverse Eurasian and North American lineages $\mathrm{H} 7$ viruses. Adult female BALB/c mice were immunized twice with DNA vaccines encoding $\mathrm{CH} 7-22, \mathrm{CH} 7-24, \mathrm{CH7}-26$, and $\mathrm{CH} 7-28$, their serum was collected and analyzed by ELISA at 2 weeks after the second immunization. As a comparison, plasmids encoding HA proteins of H7N9 and H7N7 were immunized in parallel. The serum was tested against Eurasian H7N9 and H7N7, and North American H7N3 and H7N1 viruses. The results showed that $\mathrm{CH}-22$, $\mathrm{CH}-24$, and $\mathrm{CH}-26$ elicited high antibody titers against all tested $\mathrm{H} 7$ viruses, whereas $\mathrm{CH}-28$ elicited relatively low titers against H7N3 (Figure 4). These results indicated that $\mathrm{CH} 7-22, \mathrm{CH} 7-24$, and CH7-26 induced cross-reactive anti-H7 antibodies. 

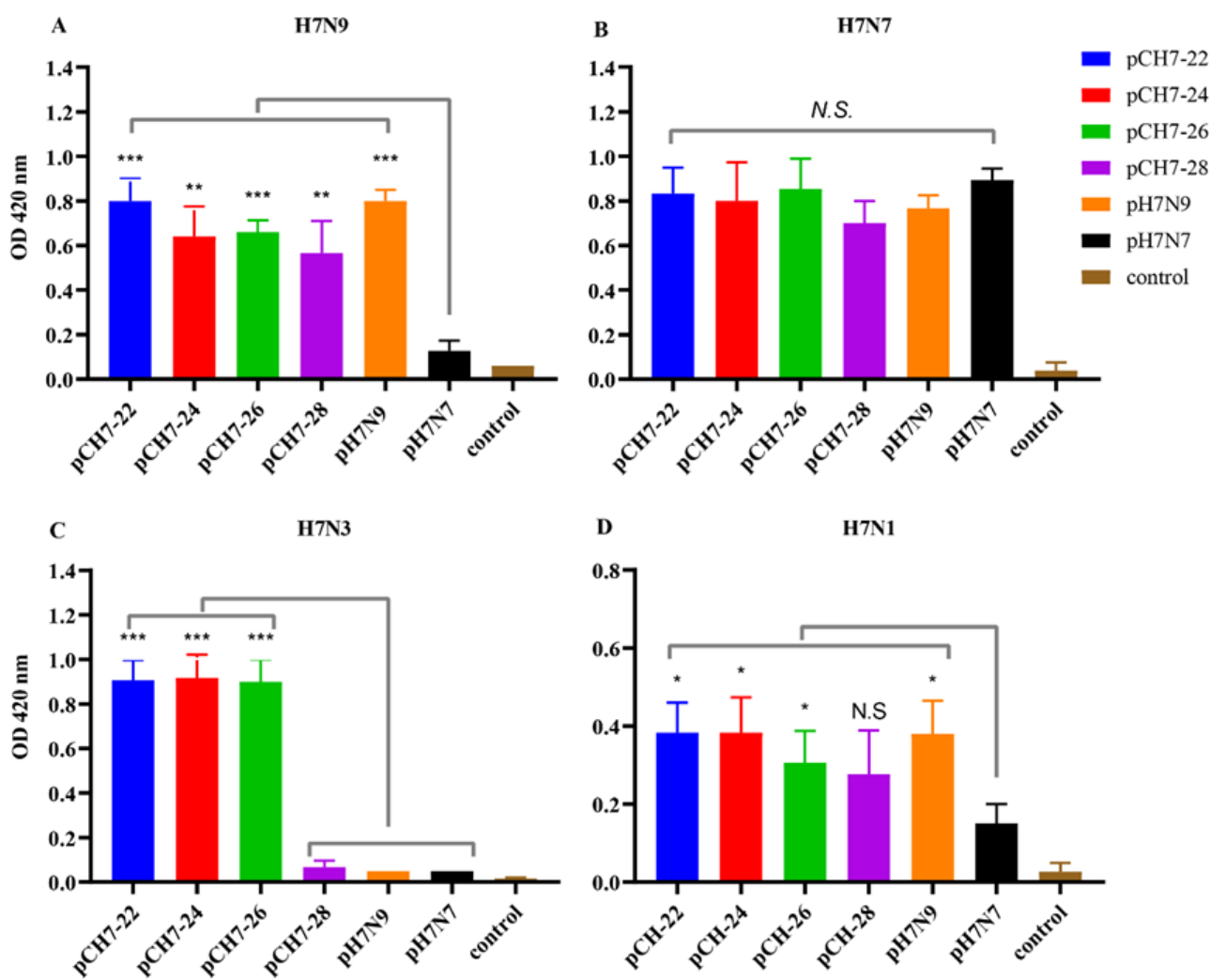

Figure 4. DNA vaccines encoding consensus $\mathrm{H} 7$ proteins elicit broadly reactive antibody responses in mice. Six to 8-week-old female BALB/c mice (10 mice per group) were immunized twice with a 3-week interval. Each vaccination consisted of $30 \mu \mathrm{g}$ pCH7-22, pCH7-24, pCH7-26, pCH7-28, pH7N9, and $\mathrm{pH} 7 \mathrm{N7}$ dissolved in $30 \mu \mathrm{L}$ Tris-EDTA buffer. Two weeks after the second immunization, sera were collected from four to five mice per group and treated with an RDE. The treated serum was tested for the titer of total IgG antibodies. An ELISA was performed using a 96-well plate coated with H7N9 (A), H7N7 (B), H7N3 (C), and H7N1 (D) (4 HAU/well), followed by incubation with serial dilutions of RDE-treated serum and then goat anti-mouse IgG ( $\gamma$-chain specific) conjugated with HRP. The amount of chromogen produced was measured at 1: 100 serum dilution based on the absorbance at $420 \mathrm{~nm}$ using an ELISA reader (Synergy H1, Biotek). The data are shown as the mean antibody titers of four mice in each group with standard errors (error bars). Statistical significance was analyzed by $t$ test. $p$ values shown in bar charts and N.S. indicates no significance between two compared groups. ${ }^{*} p<0.05,{ }^{* *} p<0.01$ and ${ }^{* * *} p<0.001$ between indicated groups.

\subsection{DNA Vaccines Encoding COBRA H7 Proteins Elicit Broadly Neutralizing Antibody Responses in Mice}

We further investigated whether the elicited antibodies had the potential to cross-neutralize H7 viruses. As shown in Figure 5, each consensus immunogen provoked effective high HAI titers against four tested viruses. Mice that received pCH7-22 had HAI titers against H7N9 of $\geq 1: 320$ and $\geq 1: 160$ titers against H7N7, H7N3, and H7N1 viruses. The mice that received pCH7-24 had titers of $\geq 1: 160$ against H7N9, H7N7, and H7N3 viruses, and $\geq 1: 80$ titers against H7N1. The HAI titer in mice vaccinated with pCH7-26 was 1:160 against H7N9, H7N3, and H7N1 viruses, and 1:320 against H7N7. Mice vaccinated with pCH7-28 had a slightly lower HAI titer against H7N3 and H7N1 (titers $\geq 1: 80$ ) compared with H7N9 and H7N7 viruses $(\geq 1: 160)$. The mice vaccinated with $p H 7 N 9$ and $p H 7 N 7$ had apparently lower cross-reactive HAI titers against heterologous strains than those against homologous viruses. Overall, these data confirmed that the DNA vaccine encoding $\mathrm{CH} 7-22, \mathrm{CH7}-24$, and $\mathrm{CH7}-26$ elicited a better broad cross-reactive HAI against divergent $\mathrm{H} 7$ viruses. Next, we examined the cross-neutralizing activity against H7N9, H7N7, H7N3, and H7N1 viruses. The results further confirmed that plasmids encoding $\mathrm{CH7}-22$, $\mathrm{CH} 7-24$, and $\mathrm{CH} 7-26$ elicited a better broad cross-reactive HAI against divergent $\mathrm{H7}$ viruses (Figure 6). 

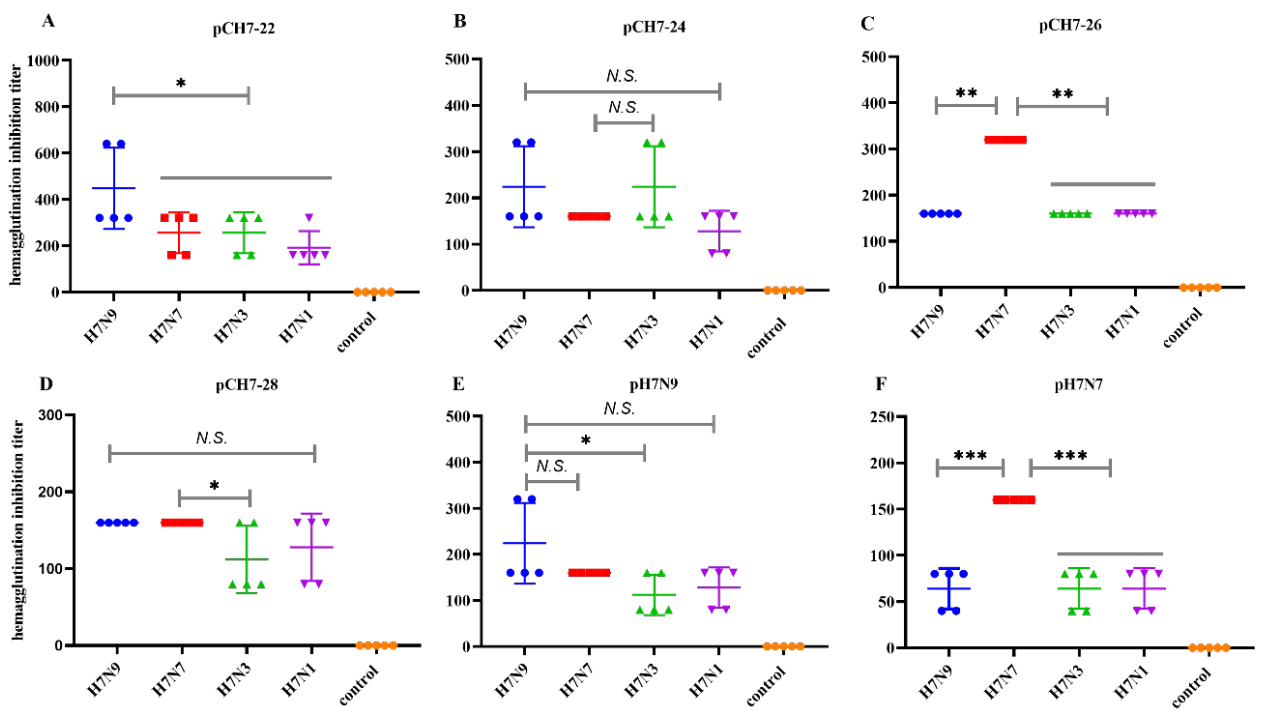

Figure 5. DNA vaccines encoding consensus $\mathrm{H} 7$ proteins elicit broad HAI antibody responses in mice. Sera from each group of immunized and unimmunized mice were serially diluted by two-fold with PBS in a 96-well polystyrene microtiter plate with $25 \mu \mathrm{L}$ in each well. A portion of $25 \mu \mathrm{L}$ virus suspension containing 4 hemagglutinin units (HAU) H7N9, H7N7, H7N3, and H7N1) were added to each well. After incubation of the plate at room temperature for $1 \mathrm{~h}, 50 \mu \mathrm{L}$ of $0.5 \%(\mathrm{v} / \mathrm{v})$ chicken red blood cells were added to each well, and the plate was incubated at room temperature for 30 minutes. HAI titers were determined as the highest serum dilution that completely inhibited hemagglutination. Values are the mean titers plus the standard error of the mean (error bars) (A-F). Statistical significance was analyzed by $t$ test or Mann-Whitney Test. $p$ values shown in bar charts and N.S. indicates no significance between two compared groups. ${ }^{*} p<0.05,{ }^{* *} p<0.01$ and ${ }^{* *} p<0.001$ between indicated groups.
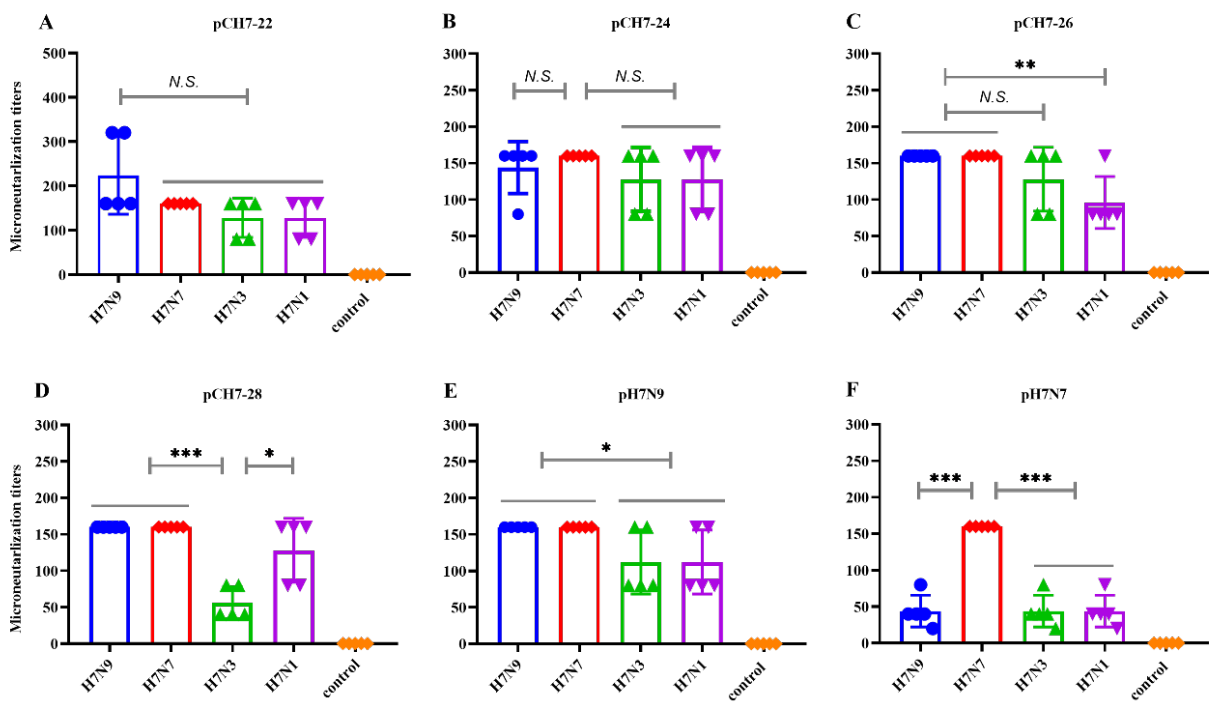

Figure 6. DNA vaccines encoding consensus $\mathrm{H7}$ proteins elicit broad neutralizing antibody responses in mice. Serum treated with a receptor-destroying enzyme (RDE) was diluted from 1:20 to 1:2560 by two-fold serial dilutions in culture medium (DMEM containing $100 \mathrm{U} / \mathrm{mL}$ penicillin $\mathrm{G}, 100 \mu \mathrm{g} / \mathrm{mL}$ streptomycin, and $0.5 \mu \mathrm{g} / \mathrm{mL}$ TPCK-treated trypsin). Diluted serum solutions were mixed with culture medium containing 100 TCID $_{50}$ H7N9, H7N7, H7N3, and H7N1 at room temperature for $1 \mathrm{~h}$. The virus-serum mix was then applied to MDCK cells. Culture medium was added, and the plates were incubated for $72 \mathrm{~h}$. Endpoints were determined by the hemagglutination titer. Values are the mean titers plus the standard error of the mean (error bars) (A-F). Statistical significance was analyzed by $t$ test. $p$ values shown in bar charts and N.S. indicates no significance between two compared groups. ${ }^{*} p<0.05,{ }^{* *} p<0.01$ and ${ }^{* * *} p<0.001$ between indicated groups. 


\subsection{DNA Vaccination with Consensuses H7 Confers Protection against Lethal H7N9 Influenza Virus Challenge in Mice}

After evaluating the cross-reactive antibody responses elicited by the consensus $\mathrm{H} 7$ against Eurasian and North American lineage H7 viruses, we investigated the efficacy of the consensus $\mathrm{H7}$ in a mouse model. Groups of mice were vaccinated twice with pCH7-22, pCH7-24, pCH7-26, pCH7-28, pH7N7, or pH7N9 and then challenged with $10 \times \mathrm{LD}_{50} \mathrm{~A} / \mathrm{Sh}$ anghai/2/2013/H7N9 virus at 3 weeks after the second immunization. Four mice from each group were sacrificed at day 5 post-infection and their lungs were collected for virus titration. Changes in body weight and survival rates were monitored daily for 2 weeks post-infection. Mice that lost more than $25 \%$ of their body weight were euthanized according to guidelines. Mice vaccinated with pCH7-22, pCH7-24, pCH7-26, pCH7-28, or pH7N9 only showed slight weight loss at $3 \mathrm{dpi}$, recovered quickly, and all survived within 2 weeks (Figure 7). Mice vaccinated with pH7N7 survived at a rate of $60 \%$ and their weight decreased significantly, reaching maximum loss at $7 \mathrm{dpi}$. Mice in the control group died within 7 days of the infection. Consistent with the survival rate and weight loss, high lung viral titers were detected in pH7N7 and control groups, whereas no virus was detected in mice vaccinated with pCH7-22, pCH7-24, pCH7-26, pCH7-28, or pH7N9 (Figure 7). These results suggest that the consensus H7 provides protection against lethal H7N9 infection in a mouse model.
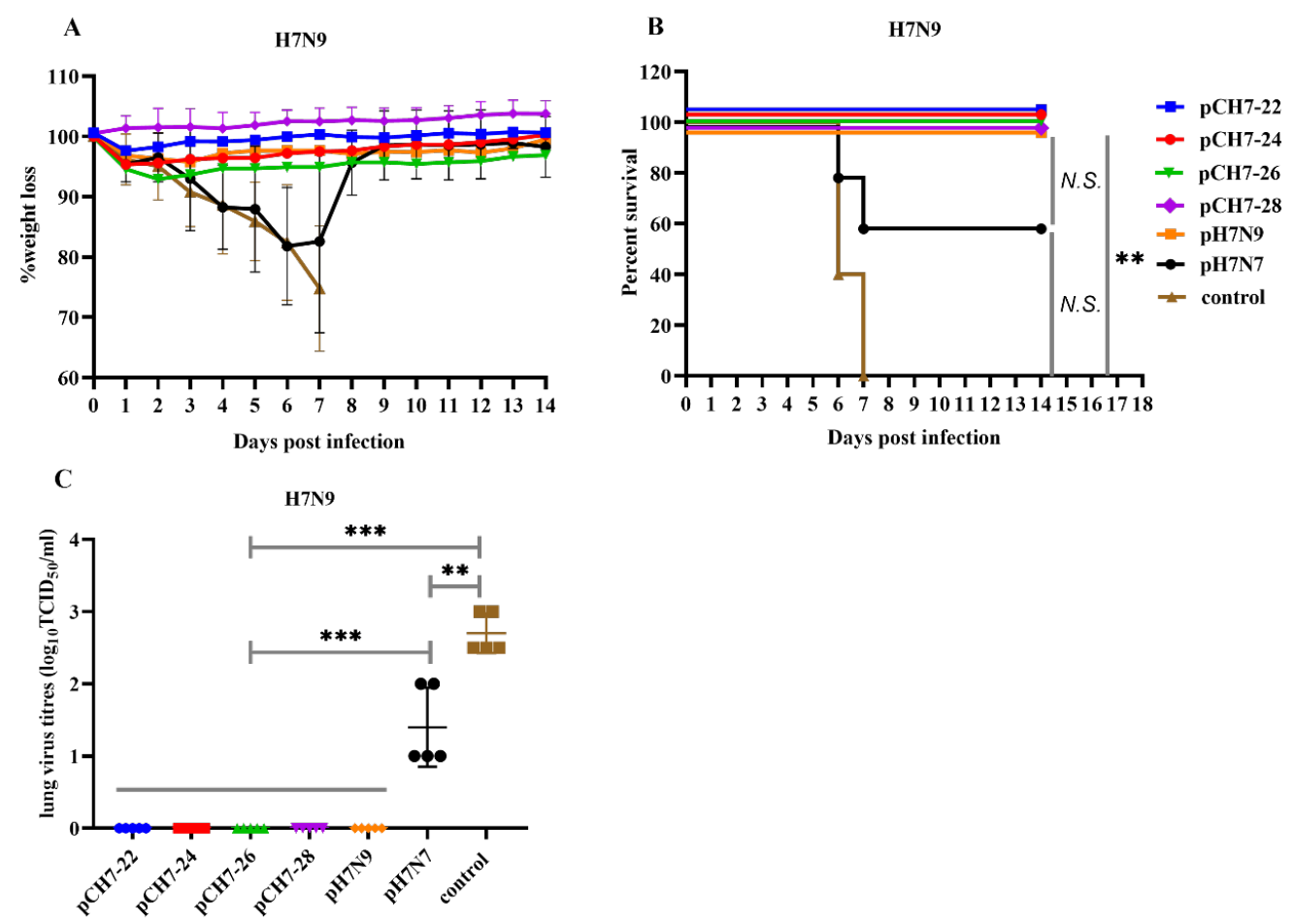

Figure 7. DNA vaccination with consensuses $\mathrm{H} 7$ confers protection against lethal H7N9 influenza virus challenge in mice. Groups of mice were vaccinated twice with pCH7-22, pCH7-24, pCH7-26, pCH7-28, pH7N7, or pH7N9 and challenged with $10 \times$ LD50 A/Shanghai/2/2013/H7N9 virus at 3 weeks after the second immunization. Changes in body weight (A) and survival rates (B) were monitored daily for 2 weeks post-infection. Four mice from each group were sacrificed at day 5 post-infection, and lungs were collected for virus titration (C). The statistical analysis of survival rate difference between groups was performed with a Fisher's Exact test. The statistical analysis of viral titers was analyzed by $t$ test. $p$ values shown in bar charts and N.S. indicates no significance between two compared groups. ** $p<0.01$ and ${ }^{* * *} p<0.001$ between indicated groups.

\subsection{DNA Vaccines Elicit Protection against H7N3 Influenza Virus Infection}

Next, avian influenza strain A/Chicken/BC/CN006/2004 (H7N3) from the North American lineage was used to test the cross-protective efficacy of pCH7-22, pCH7-24, pCH7-26, pCH7-28 pH7N7, or 
pH7N9. Mice were vaccinated as described above. At week 6, the mice were infected with a lethal dose of mouse-adapted A/Chicken/BC/CN006/2004/H7N3 $\left(10 \times \mathrm{LD}_{50}\right)$. Mice vaccinated with pCH7-22, pCH7-24, and pCH7-26 all survived and showed no symptoms of morbidity or weight loss within 2 weeks (Figure 8). However, the mice that received pCH7-28, pH7N7, and pH7N9 had apparent weight loss and only showed partial protection against lethal H7N3 infection with survival rates of $83 \%, 40 \%$, and $60 \%$, respectively (Figure 8). After 3 days of infection, body weight in the control group decreased rapidly, and all mice died at $5 \mathrm{dpi}$. Consistent with the survival rate and weight loss, no lung viruses were detected in mice vaccinated with pCH7-22 or pCH7-24 at 5 dpi. Animals vaccinated with either pCH7-26, pCH7-28-H7, pH7N7, or pH7N9 had detectable viral titers (Figure 8). However, the control group had high levels of lung viral titers compared with vaccinated groups.
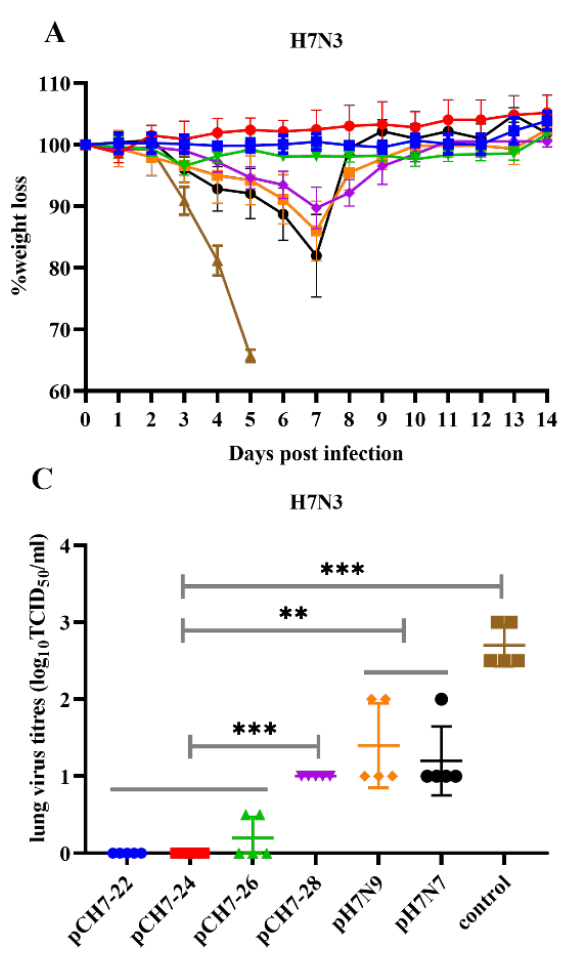

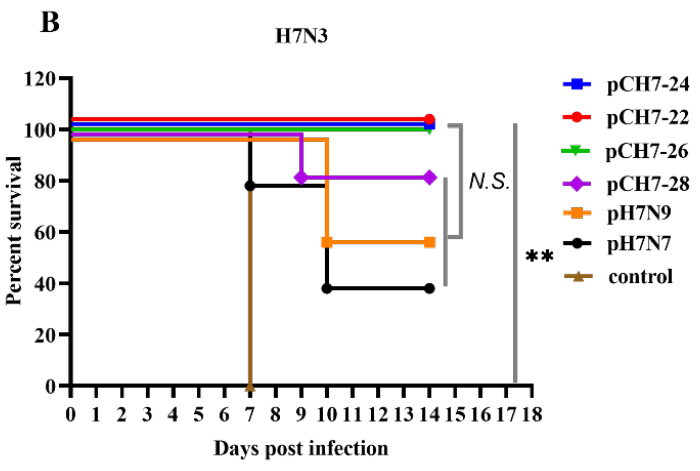

Figure 8. DNA vaccination with consensus $\mathrm{H} 7$ confers protection against lethal $\mathrm{H} 7 \mathrm{~N} 3$ influenza virus challenge in mice. Groups of mice were vaccinated twice with pCH7-22, pCH7-24, pCH7-26, pCH7-28, pH7N7, or pH7N9 and challenged with $10 \times$ LD50 mouse-adapted A/Chicken/BC/CN006/2004 (H7N3) from the North American lineage virus at 3 weeks after the second immunization. Changes in body weight (A) and survival rates (B) were monitored daily for 2 weeks post-infection. Four mice from each group were sacrificed at day 5 post-infection, and lungs were collected for virus titration (C). The statistical analysis of survival rate difference between groups was performed with a Fisher's Exact test. The statistical analysis of viral titers was analyzed by $t$ test. $p$ values shown in bar charts and N.S. indicates no significance between two compared groups. ${ }^{* *} p<0.01$ and ${ }^{* *} p<0.001$ between indicated groups.

\section{Discussion}

Vaccination is the most effective countermeasure to prevent the threat of influenza viruses. Among viral proteins of the influenza virus, HA is the main protein used as a vaccine candidate because it elicits a highly protective immune response in the host $[29,30]$. However, under selective pressure from the host immune system, evolution of HA protein antigenicity has resulted in escape of antibody recognition [29]. Consequently, yearly updated influenza vaccines consist of inactivated virus with HA from types $\mathrm{A}(\mathrm{H} 1 \mathrm{~N} 1)$ pdm09 and $\mathrm{A}(\mathrm{H} 3 \mathrm{~N} 2)$, and one/two type B that best match the predicted circulating strains, but lack cross-protection against novel variants [31]. It is currently impossible to predict which antigenic variants may emerge, and novel vaccine candidates are needed to elicit broad 
spectrum immune responses against variants. Recently, a new technology called COBRA was used to design consensus antigens of various subtypes including $\mathrm{H} 1, \mathrm{H} 3$, and $\mathrm{H} 5$. COBRA consensuses have been generated using HA protein sequences of $\mathrm{H} 5 \mathrm{~N} 1$ and vaccination elicits broad antibody responses [15]. Nine prototypes of H1N1 were evaluated in mice as virus-like particles that provoked cross-reactive immune responses against the divers of H1N1 viruses [17]. In addition, a previous study confirmed that the COBRA method is an effective strategy to develop an H3N2 universal vaccine [18]. Collectively, these findings highly suggest that COBRA is a powerful method to generate subtype universal vaccines.

In this study, four consensus $\mathrm{H7}$ antigens (CH7-22, CH7-24, CH7-26, and CH7-28) were designed by COBRA and generated. In vitro experiments confirmed that head-specific P52E03 and stem-specific CR9114 mAbs bound the cell surface-expressed consensus HAs, indicating retention of the antigenicity of the four consensus HAs. It has been shown that COBRA-designed consensus proteins retain highly immunogenic and cross-reactive epitopes [32]. Consistent with the in vitro experiments, when applied as DNA vaccines in BALB/c mice, broadly reactive antibodies against $\mathrm{H7}$ viruses from Eurasian and North American lineages were elicited and detected by inhibition, binding, and neutralizing analyses. An ideal universal influenza vaccine needs to elicit broad spectrum reactive antibodies against intra- and inter-subtype viruses. Previous studies have described that consensus HA proteins induce broadly subtype-specific neutralizing antibodies against H1, H3, and H5 HAs [15,17,18]. These results combined with our data highly suggest that using COBRA to design consensus HAs is an applicable method to develop universal vaccines for not only these subtypes, but also $\mathrm{H} 7$ and even other subtypes.

In viral infection experiments, protection against lethal H7N9 viral challenge was also provided by DNA immunization with pCH7-22, pCH7-24, pCH7-26, or pCH7-28. The viral titers were undetectable in the lungs of consensus DNA-immunized mice at 5 dpi, suggesting that the consensus H7 vaccines that we designed and tested effectively prevented lethal H7N9 infection in mice. The clinical outcomes of mice immunized with consensus $\mathrm{H} 7$ were consistent with the antibody response. The four consensus H7 DNAs induced higher IgG antibody, HAI, and neutralizing antibody titers against H7N9 than pH7N7 and induced a similar high titer compared with pH7N9. Therefore, a lower survival rate, more weight loss, and higher lung viral titers were observed in the pH7N7-immunized group. The H7N9 virus infected humans in 2013 [4], resulting in 1567 cases with at least 615 deaths as of 5 September 2018 [33]. The 2016-2017 epidemic was the fifth wave and caused the largest human infection with 758 cases and 288 deaths [34,35]. Evolutionary analysis of H7N9 viruses revealed two outbreak sources of H7N9, the Yangtze and Pearl River Delta regions [36]. Importantly, highly pathogenic H7N9 viruses emerged in the fifth wave [37]. The continuous evolution of H7N9 subtype viruses poses a long-term threat to public health and requires a universal vaccine. In the future, the consensus H7 proteins, especially CH7-22, CH7-24, and CH7-26, have the potential for development of a vaccine candidate against diversified H7N9 viruses.

We next tested the efficacy of the consensus H7 proteins against American lineage H7N3. After challenge with a lethal H7N3 virus, the results showed that all mice vaccinated with pCH7-22, pCH7-24, and pCH7-26 survived and showed no symptoms of morbidity or weight loss within 2 weeks. Consistent with the survival rate and weight loss, no lung viruses were detected in mice vaccinated with pCH7-22 or pCH7-24 at 5 dpi. Mice that received pCH7-28, pH7N7, and pH7N9 had apparent weight loss and only showed partial protection against lethal H7N3 infection with survival rates of $83 \%, 40 \%$, and $60 \%$, respectively. $\mathrm{CH} 7-28$ was derived from $\mathrm{CH} 7-26$ sequences by introducing S136N and A143V mutations based on the analysis showing that both mutations emerged in H7N9 HA since the second wave. The introduction of the two mutations may affect the conformational epitopes and subsequently result in less cross-neutralizing, since the antibody titers against American lineage H7N3 (ELISA, HAI, or MN analysis) in CH-28 immunized group were apparently lower than in the other three consensus $\mathrm{H} 7$ protein immunized groups. Based on these results, consensus $\mathrm{CH7}-22$ and CH7-24 proteins showed better efficacy against American lineage H7N3 than the other consensus 
proteins. Geographical separation of the host species has shaped H7 AIVs into independently evolving Eurasian and American lineages [38]. Among each lineage, H7 AIVs circulating in migratory birds have conserved antigenic epitopes and efficient cross-reactivity with each other. Although previous studies have shown that vaccinations with divergent heterologous $\mathrm{H} 7 \mathrm{immunogens}$ from both Eurasian and American lineages raise cross-reactive HAI antibodies against H7N9 viruses and even protect mice from lethal viral infections [39-42], few studies have described the result of immunization with a Eurasian lineage $\mathrm{H} 7$ vaccine against an American lineage virus. Here, we found that Eurasian lineage pH7N9 and pH7N7 vaccination of mice had less efficacy against American lineage H7N3 viral infection compared with consensus $\mathrm{pCH7}-22$ or $\mathrm{pCH7}-24$ proteins. These results highly suggested that both $\mathrm{CH7}-22$ and $\mathrm{CH}-24$ consensus antigens are ideal universal vaccines against divergent $\mathrm{H} 7$ viruses. In the future, we need to evaluate the efficacies of pCH7-22 and pCH7-24 against other Eurasian and American lineage H7 viruses. Furthermore, a large variety of AIVs (H5, H7, H9 and other subtypes) circulate in poultry in countries worldwide. This study will help develop universal vaccine against AIVs and contribute to prevent and control AIVs in poultry.

\section{Conclusions}

In this study, we describe development of a highly effective consensus H7 HA using the COBRA method, which stimulates robust cross-reactivate immune responses. Two (CH7-22 CH7-24) out of four consensus $\mathrm{H7}$ antigens have the potential for development as a $\mathrm{H} 7$ universal vaccine candidate. These results also highly suggest that using COBRA to design consensus HAs is an applicable method to develop universal vaccines for not only H7 subtypes, but also other subtypes (for human, poultry, or both).

Author Contributions: J.C., F.M. and G.M.F. conceived and designed the experiments; G.M.F., Z.Z., M.H., J.H., M.L. performed the experiments; H.L., B.L., Y.Y., R.G., B.Z., D.L. analyzed the data; G.M.F. and J.C. wrote the paper. All authors have read and agreed to the published version of the manuscript.

Funding: This work was funded by the National Mega Project on Major Infectious Disease Prevention [grant number 2017ZX10103005-005], National Natural Science Foundation of China [grant numbers 31570026, 31970174 and 81961138013].

Acknowledgments: We are thankful to The Core Facility and Technical Support, Wuhan Institute of Virology, Chinese Academy of Sciences (Xuefang An, Hao Tang, Yuzhou Xiao, Youling Zhu and Fan Zhang for assistance with the mice experiments).

Conflicts of Interest: The authors report no conflict of interest.

\section{References}

1. Belser, J.A.; Bridges, C.B.; Katz, J.M.; Tumpey, T.M. Past, present, and possible future human infection with influenza virus A subtype H7. Emerg. Infect. Dis. 2009, 15, 859-865. [CrossRef] [PubMed]

2. Abdelwhab, E.M.; Veits, J.; Mettenleiter, T.C. Prevalence and control of $\mathrm{H7}$ avian influenza viruses in birds and humans-ERRATUM. Epidemiol. Infect. 2014, 142, 921. [CrossRef] [PubMed]

3. Huo, X.; Cui, L.B.; Chen, C.; Wang, D.Y.; Qi, X.; Zhou, M.H.; Guo, X.L.; Wang, F.M.; Liu, W.J; Kong, W.R.; et al. Severe human infection with a novel avian-origin influenza A(H7N4) virus. Sci. Bull. 2018, 63, 1043-1050. [CrossRef]

4. Gao, R.; Cao, B.; Hu, Y.; Feng, Z.; Wang, D.; Hu, W.; Chen, J.; Jie, Z.; Qiu, H.; Xu, K.; et al. Human infection with a novel avian-origin influenza A (H7N9) virus. N. Engl. J. Med. 2013, 368, 1888-1897. [CrossRef] [PubMed]

5. Fouchier, R.A.M.; Schneeberger, P.M.; Rozendaal, F.W.; Broekman, J.M.; Kemink, S.A.G.; Munster, V.; Kuiken, T.; Rimmelzwaan, G.F.; Schutten, M.; Van Doornum, G.J.J.; et al. Avian influenza A virus (H7N7) associated with human conjunctivitis and a fatal case of acute respiratory distress syndrome. Proc. Natl. Acad. Sci. USA 2004, 101, 1356-1361. [CrossRef]

6. Li, Q.; Zhou, L.; Zhou, M.; Chen, Z.; Li, F.; Wu, H.; Xiang, N.; Chen, E.; Tang, F.; Wang, D.; et al. Epidemiology of human infections with avian influenza A(H7N9) virus in China. N. Engl. J. Med. 2014, 370, 520-532. [CrossRef] 
7. Zhu, H.; Lam, T.T.-Y.; Smith, D.K.; Guan, Y. Emergence and development of H7N9 influenza viruses in China. Curr. Opin. Virol. 2016, 16, 106-113. [CrossRef]

8. Lam, T.T.-Y.; Zhou, B.; Wang, J.; Chai, Y.; Shen, Y.; Chen, X.; Ma, C.; Hong, W.; Chen, Y.; Zhang, Y.; et al. Dissemination, divergence and establishment of H7N9 influenza viruses in China. Nature 2015, 522, $102-105$. [CrossRef]

9. Qi, W.; Jia, W.; Liu, D.; Li, J.; Bi, Y.; Xie, S.; Li, B.; Hu, T.; Du, Y.; Xing, L.; et al. Emergence and Adaptation of a Novel Highly Pathogenic H7N9 Influenza Virus in Birds and Humans from a 2013 Human-Infecting Low-Pathogenic Ancestor. J. Virol. 2018, 92. [CrossRef]

10. Lam, T.T.; Wang, J.; Shen, Y.; Zhou, B.; Duan, L.; Cheung, C.L.; Ma, C.; Lycett, S.J.; Leung, C.Y.; Chen, X.; et al. The genesis and source of the H7N9 influenza viruses causing human infections in China. Nature 2013, 502, 241-244. [CrossRef]

11. Bi, Y.; Liu, H.; Xiong, C.; Di, L.; Shi, W.; Li, M.; Liu, S.; Chen, J.; Chen, G.; Li, Y.; et al. Novel avian influenza A (H5N6) viruses isolated in migratory waterfowl before the first human case reported in China, 2014. Sci. Rep. 2016, 6, 29888. [CrossRef] [PubMed]

12. Zhou, L.; Ren, R.; Yang, L.; Bao, C.; Wu, J.; Wang, D.; Li, C.; Xiang, N.; Wang, Y.; Li, D.; et al. Sudden increase in human infection with avian influenza A(H7N9) virus in China, September-December 2016. West. Pac. Surveill. Response J. 2017, 8, 6-14. [CrossRef] [PubMed]

13. Liu, D.; Shi, W.; Shi, Y.; Wang, D.; Xiao, H.; Li, W.; Bi, Y.; Wu, Y.; Li, X.; Yan, J.; et al. Origin and diversity of novel avian influenza A H7N9 viruses causing human infection: Phylogenetic, structural, and coalescent analyses. Lancet 2013, 381, 1926-1932. [CrossRef]

14. World Health Organization. Influenza at the Human-Animal Interface. Available online: http://www.who. int/influenza/human_animal_interface/Influenza_Summary_IRA_HA_interface_07_25_2017.pdf (accessed on 25 July 2017).

15. Giles, B.M.; Ross, T.M. A computationally optimized broadly reactive antigen (COBRA) based H5N1 VLP vaccine elicits broadly reactive antibodies in mice and ferrets. Vaccine 2011, 29, 3043-3054. [CrossRef]

16. Sautto, G.A.; Kirchenbaum, G.A.; Ross, T.M. Towards a universal influenza vaccine: Different approaches for one goal. Virol. J. 2018, 15, 17. [CrossRef]

17. Carter, D.M.; Darby, C.A.; Lefoley, B.C.; Crevar, C.J.; Alefantis, T.; Oomen, R.; Anderson, S.F.; Strugnell, T.; Cortes-Garcia, G.; Vogel, T.U.; et al. Design and Characterization of a Computationally Optimized Broadly Reactive Hemagglutinin Vaccine for H1N1 Influenza Viruses. J. Virol. 2016, 90, 4720-4734. [CrossRef]

18. Wong, T.M.; Allen, J.D.; Bebin-Blackwell, A.G.; Carter, D.M.; Alefantis, T.; DiNapoli, J.; Kleanthous, H.; Ross, T.M. Computationally Optimized Broadly Reactive Hemagglutinin Elicits Hemagglutination Inhibition Antibodies against a Panel of H3N2 Influenza Virus Cocirculating Variants. J. Virol. 2017, 91. [CrossRef]

19. Comparison of several adjuvants for inactivated H7N9 influenza virus vaccine. Available online: http: //en.cnki.com.cn/Article_en/CJFDTotal-ZGYC201609009.htm (accessed on 18 March 2020).

20. Liu, H.; Xiong, C.; Chen, J.; Chen, G.; Zhang, J.; Li, Y.; Xiong, Y.; Wang, R.; Cao, Y.; Chen, Q.; et al. Two genetically diverse H7N7 avian influenza viruses isolated from migratory birds in central China. Emerg. Microbes. Infect. 2018, 7, 62. [CrossRef]

21. Li, M.; Chen, L.; Wang, Q.; Hao, M.; Zhang, X.; Liu, L.; Yu, X.; Yang, C.; Xu, J.; Chen, J.; et al. A cross-reactive human monoclonal antibody targets the conserved $\mathrm{H} 7$ antigenic site A from fifth wave H7N9-infected humans. Antivir. Res. 2019, 170, 104556. [CrossRef]

22. WHO. Serological Detection of Avian Influenza A(H7N9) Infections by Microneutralization Assay; WHO: Geneva, Switzerland, 2013.

23. Kwon, H.I.; Kim, Y.I.; Park, S.J.; Song, M.S.; Kim, E.H.; Kim, S.M.; Si, Y.J.; Lee, I.W.; Song, B.M.; Lee, Y.J.; et al. Evaluation of the Immune Responses to and Cross-Protective Efficacy of Eurasian H7 Avian Influenza Viruses. J. Virol. 2017, 91. [CrossRef]

24. Chen, J.; Liu, Q.; Chen, Q.; Xiong, C.; Yao, Y.; Wang, H.; Wang, H.; Chen, Z. Comparative analysis of antibody induction and protection against influenza virus infection by DNA immunization with HA, HAe, and HA1 in mice. Arch. Virol. 2014, 159, 689-700. [CrossRef] [PubMed]

25. Yan, J.; Yoon, H.; Kumar, S.; Ramanathan, M.P.; Corbitt, N.; Kutzler, M.; Dai, A.; Boyer, J.D.; Weiner, D.B. Enhanced cellular immune responses elicited by an engineered HIV-1 subtype B consensus-based envelope DNA vaccine. Mol. Ther. 2007, 15, 411-421. [CrossRef] [PubMed] 
26. Dreyfus, C.; Laursen, N.S.; Kwaks, T.; Zuijdgeest, D.; Khayat, R.; Ekiert, D.C.; Lee, J.H.; Metlagel, Z.; Bujny, M.V.; Jongeneelen, M.; et al. Highly conserved protective epitopes on influenza B viruses. Science 2012, 337, 1343-1348. [CrossRef] [PubMed]

27. Yao, Y.; Wang, H.; Chen, J.; Shao, Z.; He, B.; Chen, J.; Lan, J.; Chen, Q.; Chen, Z. Protection against homo and hetero-subtypic in fl uenza A virus by optimized M2e DNA vaccine. Emerg. Microbes Infect. 2019, 8, 45-54. [CrossRef] [PubMed]

28. Aihara, H.; Miyazaki, J. Gene transfer into muscle by electroporation in vivo. Nat. Biotechnol. 1998, 16, 867-870. [CrossRef] [PubMed]

29. Plotkin, J.B.; Dushoff, J. Codon bias and frequency-dependent selection on the hemagglutinin epitopes of influenza A virus. Proc. Natl. Acad. Sci. USA 2003, 100, 7152-7157. [CrossRef] [PubMed]

30. Joseph, U.; Su, Y.C.; Vijaykrishna, D.; Smith, G.J. The ecology and adaptive evolution of influenza A interspecies transmission. Influenza Other Respir. Viruses 2017, 11, 74-84. [CrossRef]

31. Berlanda Scorza, F.; Tsvetnitsky, V.; Donnelly, J.J. Universal influenza vaccines: Shifting to better vaccines. Vaccine 2016, 34, 2926-2933. [CrossRef]

32. Sautto, G.A.; Kirchenbaum, G.A.; Abreu, R.B.; Ecker, J.W.; Pierce, S.R.; Kleanthous, H.; Ross, T.M. A Computationally Optimized Broadly Reactive Antigen Subtype-Specific Influenza Vaccine Strategy Elicits Unique Potent Broadly Neutralizing Antibodies against Hemagglutinin. J. Immunol. 2020, 204, 375-385. [CrossRef]

33. World Health Organization. Human Infection with Avian Influenza A(H7N9) Virus-The Total Number of Fatal Cases is Published on a Monthly Basis by the China National Health and Family Planning Commission; World Health Organization: Geneva, Switzerland, 2018.

34. World Health Organization. 201709 Antigenic and Genetic Characteristics of Zoonotic Influenza Viruses and Development of Candidate Vaccine Viruses for Pandemic Preparedness; World Health Organization: Geneva, Switzerland, 2017.

35. Su, S.; Gu, M.; Liu, D.; Cui, J.; Gao, G.F.; Zhou, J.; Liu, X. Epidemiology, Evolution, and Pathogenesis of H7N9 Influenza Viruses in Five Epidemic Waves since 2013 in China. Trends Microbiol. 2017, 25, 713-728. [CrossRef]

36. Wang, D.; Yang, L.; Zhu, W.; Zhang, Y.; Zou, S.; Bo, H.; Gao, R.; Dong, J.; Huang, W.; Guo, J.; et al. Two Outbreak Sources of Influenza A (H7N9) Viruses Have Been Established in China. J. Virol. 2016, 90, 5561-5573. [CrossRef] [PubMed]

37. Ke, C.; Mok, C.K.P.; Zhu, W.; Zhou, H.; He, J.; Guan, W.; Wu, J.; Song, W.; Wang, D.; Liu, J.; et al. Human Infection with Highly Pathogenic Avian Influenza A(H7N9) Virus, China. Emerg. Infect. Dis. 2017, 23, 1332-1340. [CrossRef] [PubMed]

38. Obenauer, J.C.; Denson, J.; Mehta, P.K.; Su, X.; Mukatira, S.; Finkelstein, D.B.; Xu, X.; Wang, J.; Ma, J.; Fan, Y.; et al. Large-scale sequence analysis of avian influenza isolates. Science 2006, 311, 1576-1580. [CrossRef]

39. Carter, D.M.; Bloom, C.E.; Kirchenbaum, G.A.; Tsvetnitsky, V.; Isakova-Sivak, I.; Rudenko, L.; Ross, T.M. Cross-protection against H7N9 influenza strains using a live-attenuated H7N3 virus vaccine. Vaccine 2015, 33, 108-116. [CrossRef] [PubMed]

40. Goff, P.H.; Krammer, F.; Hai, R.; Seibert, C.W.; Margine, I.; Garcia-Sastre, A.; Palese, P. Induction of Cross-Reactive Antibodies to Novel H7N9 Influenza Virus by Recombinant Newcastle Disease Virus Expressing a North American Lineage H7 Subtype Hemagglutinin. J. Virol. 2013, 87, 8235-8240. [CrossRef] [PubMed]

41. Smith, G.E.; Flyer, D.C.; Raghunandan, R.; Liu, Y.; Wei, Z.P.; Wu, Y.Y.; Kpamegan, E.; Courbron, D.; Fries, L.F.; Glenn, G.M. Development of influenza H7N9 virus like particle (VLP) vaccine: Homologous A/Anhui/1/2013 (H7N9) protection and heterologous A/chicken/Jalisco/CPA1/2012 (H7N3) cross-protection in vaccinated mice challenged with H7N9 virus. Vaccine 2013, 31, 4305-4313. [CrossRef] [PubMed]

42. Xu, Q.; Chen, Z.Y.; Cheng, X.; Xu, L.; Jin, H. Evaluation of Live Attenuated H7N3 and H7N7 Vaccine Viruses for Their Receptor Binding Preferences, Immunogenicity in Ferrets and Cross Reactivity to the Novel H7N9 Virus. PLoS ONE 2013, 8, e76884. [CrossRef]

(C) 2020 by the authors. Licensee MDPI, Basel, Switzerland. This article is an open access article distributed under the terms and conditions of the Creative Commons Attribution (CC BY) license (http://creativecommons.org/licenses/by/4.0/). 